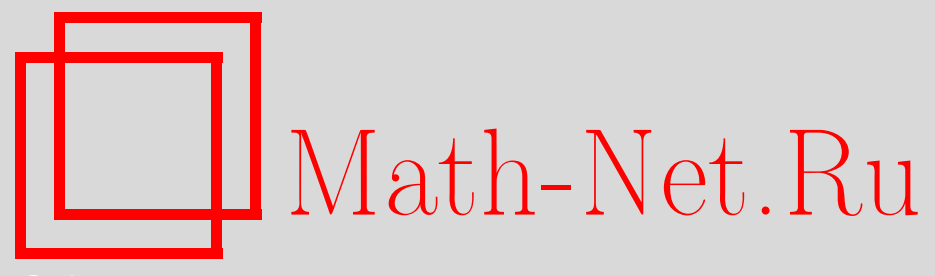

И. Д. Кан, Уточнение правила сравнения континуантов, Дискрет. матем., 2000, том 12, выпуск 3, 72-75

DOI: https://doi.org/10.4213/dm341

Использование Общероссийского математического портала Math-Net.Ru подразумевает, что вы прочитали и согласны с пользовательским соглашением http://www.mathnet.ru/rus/agreement

Параметры загрузки:

IP: 18.234 .156 .22

26 апреля 2023 г., 14:34:48 


\title{
Уточнение правила сравнения континуантов
}

\author{
(c) 2000 г. И. Д. Кан
}

\begin{abstract}
Доказывается теорема, позволяющая в ряде случаев сравнивать значения континуантов знаменателей конечных цепных дробей, не прибегая к их вычислению.
\end{abstract}

Для натуральных чисел $a_{1}, a_{2}, \ldots, a_{n}$ через $\left[a_{1}, a_{2}, \ldots, a_{n}\right]$ обозначим цепную дробь

$$
\left.\alpha=1 /\left(a_{1}+1 /\left(a_{2}+\ldots+1 / a_{n}\right)\right) \ldots\right),
$$

a через $\left\langle a_{1}, a_{2}, \ldots, a_{n}\right\rangle$ - ее знаменатель, называемый континуантом чисел

$$
a_{1}, a_{2}, \ldots, a_{n}
$$

Тогда числитель дроби $\alpha$ равен $\left\langle a_{2}, a_{3}, \ldots, a_{n}\right\rangle$ (см. книгу А. Я. Хинчина [1]). Континуант может быть также определен индуктивно: если положить

$$
x_{0}=1, \quad x_{1}=a_{1}, x_{j+1}=a_{j+1} x_{j}+x_{j-1}, \quad 1 \leqslant j<n,
$$

ro

$$
\left\langle a_{1}, a_{2}, \ldots, a_{n}\right\rangle=x_{n} .
$$

Для пустой последовательности $\varnothing$ обычно полагают $[\varnothing]=0,\langle\varnothing\rangle=1$.

Начало изучения континуантов положил Эйлер [2]. Проблемы, связанные с континуантами, актуальны и в наше время: так, недавно вышедшая книга [3] (см. стр. 333-352) содержит ряд конкретных формул и приложений континуантов. Однако о сравнении континуантов известно немного.

Информация о том, в каких нетривиальных случаях можно непосредственно узнать, который из двух континуантов больше, не вычисляя их значений, содержится в лемме статьи [4]. Именно, если для натуральных $b, c, e, f$ выполнено неравенство

$$
(b-f)(c-e)>0
$$

To

$$
\langle\vec{a}, b, c, \vec{d}, e, f, \vec{g}\rangle \geqslant\langle\vec{a}, b, e, \overleftarrow{d}, c, f, \vec{g}\rangle
$$

где, здесь и далее, переменная без стрелки - натуральное число, переменная со стрелкой (вправо или влево) - последовательность натуральных чисел (возможно 
пустая), переменная со стрелкой влево по отношению к той же переменной со стрелкой вправо обозначает последовательность, записанную в обратном порядке. Так, если

$$
\vec{d}=\left\{d_{1}, d_{2}, \ldots, d_{k-1}, d_{k}\right\}
$$

To

$$
\overleftarrow{d}=\left\{d_{k}, d_{k-1}, \ldots, d_{2}, d_{1}\right\}
$$

и наоборот.

В настоящей статье доказана следующая теорема, обобщающая правило (1), (2), вплоть до критерия выполнения равенства (2).

Теорема 1. Неравенство

$$
\langle\vec{p}, \vec{m}, \vec{q}\rangle \geqslant\langle\vec{p}, \overleftarrow{m}, \vec{q}\rangle
$$

выполнено тогда и толъко тогда, когда

$$
([\overleftarrow{p}]-[\vec{p}])([\vec{m}]-[\overleftarrow{m}]) \geqslant 0
$$

Кроме того, неравенства (3) и (4) могут обращатъся в равенства толъко одновременно.

Доказательству теоремы предпошлем ряд замечаний. Прежде всего отметим, что, как известно [1], для сравнения цепных дробей вычислений не требуется. Так, результат сравнения цепных дробей $\left[y_{1}, y_{2}, \ldots, y_{n}\right]$ и $\left[z_{1}, z_{2}, \ldots, z_{k}\right]$ зависит, в общих чертах, от четности минимального индекса $j$ такого, что $y_{j} \neq z_{j}$, и от знака разности $y_{j}-z_{j}$. Если, скажем, $y_{1}>z_{1}$, то

$$
\left[y_{1}, y_{2}, \ldots, y_{n}\right] \leqslant\left[z_{1}, z_{2}, \ldots, z_{k}\right]
$$

Равенство здесь возможно, только если одновременно выполнен ряд условий: $y_{1}=z_{1}+1, z_{2}=1, n=1, r=2$.

Следовательно, импликация неравенств $(1) \Rightarrow(2)$ вытекает из теоремы 1 при подстановках

$$
\vec{p}=(\vec{a}, b), \quad \vec{m}=(c, \vec{d}, e), \quad \vec{q}=(f, \vec{g})
$$

С другой стороны, применение правила (1), (2) к задаче о вычислении минимума в множестве континуантов, составленных из четырех единиц и четырех двоек, дает два кандидата на решение: это континуанты

$$
\langle 1,2,1,2,1,2,2,1\rangle, \quad\langle 1,2,1,2,2,1,2,1\rangle \text {, }
$$

не сравнимые в рамках метода. Между тем, положив $\vec{m}=\{2,1,2,2\}$ и применив теорему 1 , получаем, что

$$
\langle 1,2,1, \vec{m}, 1\rangle>\langle 1,2,1, \overleftarrow{m}, 1\rangle
$$

и минимум интересующего нас множества равен

$$
\langle 1,2,1,2,2,1,2,1\rangle \text {. }
$$


В силу сказанного, теорема 1 сильнее правила (1), (2).

Более сложные примеры использования теоремы 1 основаны на следующем соображении. Если $\pi$ - циклическая перестановка индексов $1, \ldots, n, n \geqslant 3$, то неравенства

$$
\left\langle a_{1}, a_{2}, \ldots, a_{n}\right\rangle \leqslant\left\langle a_{\pi(1)}, a_{\pi(2)}, \ldots, a_{\pi(n)}\right\rangle
$$

и

$$
\left\langle a_{2}, a_{3}, \ldots, a_{n-1}\right\rangle \geqslant\left\langle a_{\pi(2)}, a_{\pi(3)}, \ldots, a_{\pi(n-1)}\right\rangle
$$

могут быть выполнены или не выполнены только одновременно (в том числе как равенства). Это следует из того, что сумма левых частей двух последних неравенств представляет собой след матричного произведения

$$
\left(\begin{array}{cc}
0 & 1 \\
1 & a_{1}
\end{array}\right)\left(\begin{array}{cc}
0 & 1 \\
1 & a_{2}
\end{array}\right) \cdots\left(\begin{array}{cc}
0 & 1 \\
1 & a_{n}
\end{array}\right)
$$

(см. [3, стр. 351, упр.87]), инвариантный относительно циклических перестановок матриц-сомножителей.

Пример 1. Для сравнения континуантов

$$
\langle 1,2,2,1,1,2,1,2\rangle, \quad\langle 1,1,2,1,2,1,2,2\rangle
$$

связанных некоторой циклической перестановкой, рассмотрим континуанты

$$
\langle 2,2,1,1,2,1\rangle, \quad\langle 1,2,1,2,1,2\rangle \text {. }
$$

Полагая $\vec{m}=(2,1)$ и используя теорему 1 , находим, что

$$
\begin{aligned}
\langle 2,2,1,1,2,1\rangle & =\langle 2, \vec{m}, 1,2,1\rangle\rangle\langle 2, \overleftarrow{m}, 1,2,1\rangle \\
& =\langle 1,2,1, \vec{m}=(2,1), 2\rangle=\langle 1,2,1,2,1,2\rangle
\end{aligned}
$$

здесь в выкладке порядок записи элементов континуанта был заменен на противоположный. Отсюда, в силу сказанного выше о циклических перестановках в континуантах, следует, что

$$
\langle 1,2,2,1,1,2,1,2\rangle<\langle 1,1,2,1,2,1,2,2\rangle \text {. }
$$

Доказательство теоремъ 1. Очевидно, что достаточно рассмотреть случай $\vec{m} \neq$ $\overleftarrow{m}$, для которого последовательность $\vec{m}$ состоит не менее, чем из двух элементов. Если каждая из последовательностей $\vec{p}$ и $\overleftarrow{q}$ непуста, то, полагая, что $\vec{m}, \vec{p}$ и $\vec{q}$ имеют вид (5), и применяя к вычислению континуантов правило азбуки Морзе (см. $[3$, стр. 334]), находим, что

$$
\begin{aligned}
& \langle\vec{p}, \vec{m}, \vec{q}\rangle=\langle\vec{p}\rangle\langle\vec{m}\rangle\langle\vec{q}\rangle+\langle\vec{a}\rangle\langle\vec{d}\rangle\langle\vec{g}\rangle+\langle\vec{a}\rangle\langle\vec{d}, e\rangle\langle\vec{q}\rangle+\langle\vec{p}\rangle\langle c, \vec{d}\rangle\langle\vec{g}\rangle \\
& \langle\vec{p}, \overleftarrow{m}, \vec{q}\rangle=\langle\vec{p}\rangle\langle\vec{m}\rangle\langle\vec{q}\rangle+\langle\vec{a}\rangle\langle\vec{d}\rangle\langle\vec{g}\rangle+\langle\vec{a}\rangle\langle c, \vec{d}\rangle\langle\vec{q}\rangle+\langle\vec{p}\rangle\langle\vec{d}, e\rangle\langle\vec{g}\rangle
\end{aligned}
$$

откуда

$$
\langle\vec{p}, \vec{m}, \vec{q}\rangle-\langle\vec{p}, \overleftarrow{m}, \vec{q}\rangle=(\langle\vec{a}\rangle\langle\vec{q}\rangle-\langle\vec{p}\rangle\langle\vec{q}\rangle)(\langle\vec{d}, e\rangle-\langle c, \vec{d}\rangle)
$$


(разложение этой разности на множители содержится в [4]). Проводя деление обеих частей последнего равенства на $\langle\vec{p}\rangle\langle\vec{m}\rangle\langle\vec{q}\rangle$ получаем, что

$$
\frac{\langle\vec{p}, \vec{m}, \vec{q}\rangle-\langle\vec{p}, \overleftarrow{m}, \vec{q}\rangle}{\langle\vec{p}\rangle\langle\vec{m}\rangle\langle\vec{q}\rangle}=([\overleftarrow{p}]-[\vec{q}])([\vec{m}]-[\overleftarrow{m}])
$$

Аналогичный вывод этого равенства при $\vec{p}=\varnothing$ или $\vec{q}=\varnothing$ оставляем читателю.

Теорема доказана.

Теорема 1 является скорее инструментом, чем конечным результатом. Для формулировки следствия введем следующие обозначения. Пусть $\vec{r}=\left(r_{1}, r_{2}, \ldots, r_{n}\right)$, $V_{n}(\vec{r})$ - множество континуантов последовательностей, начинающихся с единицы и состоящих из $r_{j}$ раз встречающихся чисел $j, j=1, \ldots, n$.

Следствие 1. Справедливо равенство

$$
\max V_{n}(\vec{r})=\langle 1, \ldots, 1,2, \ldots, 2, \ldots, n, \ldots, n\rangle,
$$

где число $j$ встречаетсл ровно $r_{j}$ раз, $j=1, \ldots, n$.

\section{Список литературы}

1. Хинчин А. Я., Цепнъе дроби. Физматгиз, Москва, 1961.

2. Eulero L., Specimen algorithmi singularis. Opera Omnia (1762) 15, 31-49.

3. Грехем Р., Кнут Д., Паташник О., Кояхретная математиха. Основание информатихи. Мир, Москва, 1998.

4. Motzkin T. S., Straus E. G., Some combinatorial extremum problems. Proc. Amer. Math. Soc. (1956) 7, 1014-1021.

Статья поступила 09.0=6.1999. 